\title{
Applications of the Complex Exponential Integral
}

\section{By Murlan S. Corrington}

1. Introduction. The recent publication of an extensive table of the exponential integral for complex arguments [1] makes it possible to evaluate a large number of indefinite integrals not in existing tables, and to obtain values for the sine and cosine integrals for complex arguments.

2. Definition of Exponential Integral. The definition used by the National Bureau of Standards will be used throughout,

$$
E_{1}(z)=\int_{z}^{\infty} \frac{e^{-u}}{u} d u=R E_{1}(z)+i I E_{1}(z)
$$

where $z=x+i y . R$ and $I$ denote the real and imaginary components respectively. The integral converges if the upper limit is $\infty e^{i \alpha}$, and is independent of $\alpha$, so long as $-\frac{1}{2} \pi \leqq \alpha \leqq \frac{1}{2} \pi$ [2]. To make $E_{1}(z)$ a single-valued function a branch cut is made just below the negative real axis, including the origin, such that $z=x+i y=$ $\rho e^{i \theta},-\pi<\theta \leqq \pi$. This means that when the integral is evaluated for a point on the negative real axis the contour must be indented above the pole at the origin.

The values of $E_{1}(z)$ are given in the tables for the region $0<\theta \leqq \pi$; those for the region $-\pi<\theta<0$ can be obtained from the relations

$$
E_{1}(\bar{z})=\overline{E_{1}(z)}, \quad \bar{z}=x-i y .
$$

Here, as usual, $\overline{f(z)}$ means the complex conjugate of $f(z)$.

3. Relation to Exponential Integral for Real Arguments. The earlier tables of the exponential integral [3] for real arguments use the following definitions:

$$
E i(x)=\oint_{-\infty}^{\infty} \frac{e^{u}}{u} d u=-\oint_{-x}^{\infty} \frac{e^{-u}}{u} d u
$$

and

$$
-E i(-x)=\int_{x}^{\infty} \frac{e^{-u}}{u} d u,
$$

where $\mathscr{f}$ is the Cauchy principal value. This gives the relations

$$
E_{1}(y)=-E i(-y)
$$$$
y>0
$$

and

$$
-E_{1}(-y)=E i(y)+i \pi
$$

4. Sine and Cosine Integrals for Complex Arguments. Let $u=i t$, then equation (1) becomes

Received January 8, 1960; revised June 20, 1960. 


$$
\begin{aligned}
E_{1}(x+i y) & =\int_{y-i x}^{\infty} \frac{e^{-i t}}{t} d t \\
& =\int_{y-i x}^{\infty} \frac{\cos t}{t} d t-i \int_{y-i x}^{\infty} \frac{\sin t}{t} d t \\
& =-C i(y-i x)-i\left[\frac{1}{2} \pi-S i(y-i x)\right] .
\end{aligned}
$$

Interchanging letters gives the relations

$$
E_{1}(y-i x)=-C i(x+i y)+i\left[\frac{1}{2} \pi-S i(x+i y)\right]
$$

and

$$
E_{1}(-y+i x)=-C i(x+i y)-i\left[\frac{1}{2} \pi-S i(x+i y)\right] .
$$

Subtracting equations (7) and (8) gives

$$
\begin{aligned}
S i(x+i y)= & \frac{1}{2} i\left[\overline{E_{1}(y+i x)}-E_{1}(-y+i x)\right]+\frac{1}{2} \pi \\
= & \frac{1}{2}\left[I E_{1}(y+i x)+I E_{1}(-y+i x)+\pi\right] \\
& +\frac{1}{2} i\left[R E_{1}(y+i x)-R E_{1}(-y+i x)\right] .
\end{aligned}
$$

Adding equations ( 7 ) and (8) gives

$$
\begin{aligned}
C i(x+i y)=-\frac{1}{2}\left[R E_{1}(y+i x)+\right. & \left.R E_{1}(-y+i x)\right] \\
& +\frac{1}{2} i\left[I E_{1}(y+i x)-I E_{1}(-y+i x)\right] .
\end{aligned}
$$

In the other quadrants the following relations can be used:

$$
\begin{aligned}
S i(x-i y) & =\overline{S i(x+i y)}, \\
S i(-x+i y) & =-\overline{S i(x+i y)}, \\
S i(-x-i y) & =S i(x+i y), \\
C i(x-i y) & =\overline{C i(x+i y)}, \\
C i(-x+i y) & =\overline{C i(x+i y)}+i \pi, \\
C i(-x-i y) & =C i(x+i y)-i \pi .
\end{aligned}
$$

When $x=0$ in equation (6)

$$
E_{1}(i y)=-C i(y)-i\left[\frac{1}{2} \pi-S i(y)\right],
$$

so

$$
S i(y)=\frac{1}{2} \pi+I E_{1}(i y)
$$

and

$$
C i(y)=-R E_{1}(i y) .
$$

5. Relation to Previously Published Tables. Tables of sine and cosine integrals for complex arguments in rectangular coordinates have been published by Bleick [4]. His definition of the sine integral is not the one given here, and his function is not an analytic function. Since he defined the sine integral as 


$$
S i_{B}(x+i y)=\int_{i v}^{x+i y} \frac{\sin t}{t} d t
$$

the relation to the one used here is

$$
\begin{aligned}
S i(x+i y) & =S i(i y)+S i_{B}(x+i y) \\
& =S i_{B}(x+i y)+\frac{1}{2} i\left[E_{1}(y)-E_{1}(-y)\right]+\frac{1}{2} \pi .
\end{aligned}
$$

His definition of the cosine integral leads to the same values as the one here.

A table of $E_{1}(z)$ for arguments in polar coordinates has been published by Mashiko for angles in the first quadrant [5]. Computer routines for the complex exponential integral are also available [6].

6. Table of Integrals. The following integrals, in which $a$ and $b$ are real, can be evaluated in terms of the real and imaginary components of the exponential integral:

$$
\begin{aligned}
& \int_{0}^{b} \frac{\sin t}{a^{2}+t^{2}} d t=\frac{e^{a}}{2 a}\left[R E_{1}(a)-R E_{1}(a+i b)\right] \\
& -\frac{e^{-a}}{2 a}\left[R E_{1}(-a)-R E_{1}(-a+i b)\right], \\
& \int_{0}^{b} \frac{\cos t}{a^{2}+t^{2}} d t=\frac{e^{-a}}{2 a}\left[\pi+I E_{1}(-a+i b)\right]-\frac{e^{a}}{2 a} I E_{1}(a+i b), \\
& \int_{0}^{b} \frac{t \sin t}{a^{2}+t^{2}} d t=\frac{1}{2} e^{-a}\left[\pi+I E_{1}(-a+i b)\right]+\frac{1}{2} e^{a} I E_{1}(a+i b), \\
& \int_{0}^{b} \frac{t \cos t}{a^{2}+t^{2}} d t=\frac{1}{2} e^{a}\left[R E_{1}(a)-R E_{1}(a+i b)\right] \\
& +\frac{1}{2} e^{-a}\left[R E_{1}(-a)-R E_{1}(-a+i b)\right], \\
& \int_{0}^{b} \frac{\sinh t}{a^{2}+t^{2}} d t=-\frac{\sin a}{2 a}\left[R E_{1}(b+i a)+R E_{1}(-b+i a)+2 C i(a)\right] \\
& -\frac{\cos a}{2 a}\left[I E_{1}(b+i a)+I E_{1}(-b+i a)-2 S i(a)+\pi\right], \\
& \int_{0}^{b} \frac{\cosh t}{a^{2}+t^{2}} d t=\frac{\sin a}{2 a}\left[R E_{1}(b+i a)-R E_{1}(-b+i a)\right] \\
& +\frac{\cos a}{2 a}\left[I E_{1}(b+i a)-I E_{1}(-b+i a)\right], \\
& \int_{0}^{b} \frac{t \sinh t}{a^{2}+t^{2}} d t=\frac{1}{2} \cos a\left[R E_{1}(b+i a)-R E_{1}(-b+i a)\right] \\
& -\frac{1}{2} \sin a\left[I E_{1}(b+i a)-I E_{1}(-b+i a)\right], \\
& \int_{0}^{b} \frac{t \cosh t}{a^{2}+t^{2}} d t=\frac{1}{2} \sin a\left[I E_{1}(b+i a)+I E_{1}(-b+i a)-2 \operatorname{Si}(a)+\pi\right] \\
& -\frac{1}{2} \cos a\left[R E_{1}(b+i a)+R E_{1}(-b+i a)+2 C i(a)\right] \text {, } \\
& \int_{0}^{b} \frac{e^{t}}{a^{2}+t^{2}} d t=-\frac{\sin a}{a}\left[R E_{1}(-b+i a)+C i(a)\right] \\
& -\frac{\cos a}{a}\left[I E_{1}(-b+i a)-S i(a)+\frac{1}{2} \pi\right] \text {, }
\end{aligned}
$$




$$
\begin{aligned}
\int_{0}^{b} \frac{e^{-t}}{a^{2}+t^{2}} d t=\frac{\sin a}{a}\left[R E_{1}(b+i a)\right. & +C i(a)] \\
& +\frac{\cos a}{a}\left[I E_{1}(b+i a)-S i(a)+\frac{1}{2} \pi\right],
\end{aligned}
$$

$$
\begin{aligned}
\int_{0}^{b} \frac{t e^{t}}{a^{2}+t^{2}} d t=\sin a\left[I E_{1}(-b+i a)\right. & \left.-S i(a)+\frac{1}{2} \pi\right] \\
& -\cos a\left[R E_{1}(-b+i a)+C i(a)\right],
\end{aligned}
$$

$$
\begin{aligned}
\int_{0}^{b} \frac{t e^{-t}}{a^{2}+t^{2}} d t=\sin a\left[I E_{1}(b+i a)-S i(a)\right. & \left.+\frac{1}{2} \pi\right] \\
& -\cos a\left[R E_{1}(b+i a)+C i(a)\right],
\end{aligned}
$$

(29) $\int_{0}^{b} \frac{\sinh t}{t} d t=\frac{1}{2}\left[R E_{1}(b)-R E_{1}(-b)\right]$,

(30) $\int_{0}^{b} \frac{1-\cosh t}{t} d t=\log \gamma b+\frac{1}{2}\left[R E_{1}(b)+R E_{1}(-b)\right]$,

$$
\begin{aligned}
\int_{0}^{1} \frac{1-\cos a t \cosh b t}{t} d t=\log \gamma+ & \frac{1}{2} \log \left(a^{2}+b^{2}\right) \\
& +\frac{1}{2}\left[R E_{1}(b+i a)+R E_{1}(-b+i a)\right]
\end{aligned}
$$

where log $\gamma=0.5772156649 \cdots=$ Euler's constant,

$$
\int_{0}^{1} \frac{\sin a t \sinh b t}{t} d t=\tan ^{-1} \frac{b}{a}-\frac{1}{2}\left[I E_{1}(b+i a)-I E_{1}(-b+i a)\right],
$$$$
\int_{0}^{1} \frac{\cos a t \sinh b t}{t} d t=\frac{1}{2}\left[R E_{1}(b+i a)-R E_{1}(-b+i a)\right],
$$$$
\int_{0}^{1} \frac{\sin a t \cosh b t}{t} d t=\frac{1}{2}\left[I E_{1}(b+i a)+I E_{1}(-b+i a)+\pi\right] \text {, }
$$$$
\int_{0}^{b} \sin t \log \left(a^{2}+t^{2}\right) d t=\log a^{2}-\cos b \log \left(a^{2}+b^{2}\right)
$$$$
+e^{a}\left[R E_{1}(a)-R E_{1}(a+i b)\right]+e^{-a}\left[R E_{1}(-a)-R E_{1}(-a+i b)\right] \text {, }
$$

$\int_{0}^{b} \cos t \log \left(a^{2}+t^{2}\right) d t=\sin b \log \left(a^{2}+b^{2}\right)$

$$
-e^{-a}\left[I E_{1}(-a+i b)+\pi\right]-e^{a} I E_{1}(a+i b),
$$

$\int_{0}^{b} \sinh t \log \left(a^{2}+t^{2}\right) d t=\cosh b \log \left(a^{2}+b^{2}\right)-\log a^{2}$

$$
\begin{aligned}
-\sin a\left[I E_{1}(b+i a)+I E_{1}(-b+i a)-2 S i(a)+\pi\right] \\
+\cos a\left[R E_{1}(b+i a)+R E_{1}(-b+i a)+2 C i(a)\right],
\end{aligned}
$$

$$
\begin{aligned}
\int_{0}^{b} \cosh t \log \left(a^{2}+t^{2}\right) d t=\sinh b & \log \left(a^{2}+b^{2}\right) \\
+\sin a\left[I E_{1}(b+i a)\right. & \left.-I E_{1}(-b+i a)\right] \\
& -\cos a\left[R E_{1}(b+i a)-R E_{1}(-b+i a)\right],
\end{aligned}
$$




$$
\begin{aligned}
& \int_{0}^{b} \sin t \tan ^{-1} \frac{t}{a} d t=-\cos b \tan ^{-1} \frac{b}{a}-\frac{e^{a}}{2} I E_{1}(a+i b) \\
& +\frac{e^{-a}}{2}\left[\pi+\left[E_{1}(-a+i b)\right],\right. \\
& \int_{0}^{b} \cos t \tan ^{-1} \frac{t}{a} d t=\sin b \tan ^{-1} \frac{b}{a}+\frac{e^{i}}{2}\left[R E_{1}(a+i b)-R E_{1}(a)\right] \\
& -\frac{e^{-a}}{2}\left[R E_{1}(-a+i b)-R E_{1}(-a)\right],
\end{aligned}
$$

$$
\begin{array}{r}
\int_{0}^{b} \sinh t \tan ^{-1} \frac{t}{a} d t=\cosh b \tan ^{-1} \frac{b}{a}-\frac{\sin a}{2}\left[R E_{1}(b+i a)-R E_{1}(-b+i a)\right] \\
-\frac{\cos a}{2}\left[I E_{1}(b+i a)-I E_{1}(-b+i a)\right], \\
\int_{0}^{b} \cosh t \tan ^{-1} \frac{t}{a} d t=\sinh b \tan ^{-1} \frac{b}{a}+\sin a C i(a)+\cos a\left[\frac{1}{2} \pi-S i(a)\right]
\end{array}
$$$$
+\frac{\sin a}{2}\left[R E_{1}(b+i a)+R E_{1}(-b+i a)\right]
$$$$
+\frac{\cos a}{2}\left[I E_{1}(b+i a)+I E_{1}(-b+i a)\right],
$$

$$
\begin{array}{cc}
\int_{0}^{1} \frac{e^{a t} \sin b t}{t} d t=-\tan ^{-1} \frac{b}{a}+I E_{1}(-a+i b)+\pi, & a>0, \\
\int_{0}^{1} \frac{e^{-a t} \sin b t}{t} d t=\tan ^{-1} \frac{b}{a}+I E_{1}(a+i b), & a>0, \\
\int_{0}^{1} \frac{e^{-a t}(1-\cos b t)}{t} d t=\frac{1}{2} \log \left(1+\frac{b^{2}}{a^{2}}\right)-R E_{1}(a)+R E_{1}(a+i b), & +2 \cos a\left[R E_{1}(-b+i a)+C i(a)\right], \\
\int_{0}^{b} e^{t} \log \left(a^{2}+t^{2}\right) d t=e^{b} \log \left(a^{2}+b^{2}\right)-\log a^{2} & \\
-2 \sin a\left[I E_{1}(-b+i a)-S i(a)+\frac{1}{2} \pi\right] & \\
\int_{0}^{b} e^{t} \tan ^{-1} \frac{t}{a} d t=e^{b} \tan ^{-1} \frac{b}{a}+\sin a\left[R E_{1}(-b+i a)+C i(a)\right] & +\cos a\left[I E_{1}(-b+i a)-S i(a)+\frac{1}{2} \pi\right] .
\end{array}
$$

The integrals of equations (19) and (20) have been tabulated by Bleick [ $t]$.

7. Extension of the Table of Integrals. Other more complex integrals can be derived from the above table by differentiation or integration with respect to $a$ or $b$, using the Cauchy-Riemann relations,

$$
\begin{aligned}
\frac{\partial R E_{1}(a+i b)}{\partial a} & =\frac{\partial I E_{1}(a+i b)}{\partial b} \\
& =\frac{e^{-a}}{a^{2}+b^{2}}[b \sin b-a \cos b]
\end{aligned}
$$


and

(49)

$$
\begin{aligned}
\frac{\partial R E_{1}(a+i b)}{\partial b} & =-\frac{\partial I E_{1}(a+i b)}{\partial a} \\
& =\frac{e^{-a}}{a^{2}+b^{2}}[-a \sin b-b \cos b] .
\end{aligned}
$$

Defense Electronic Products Iivision

Radio Corporation of America

Camden, New Jersey

1. Nat. Bur. Standards, Appl. Math. Ser. No. 51, Tables of the Exponential Integral for Complex Arguments, U. S. Government Printing Office, Washington 25, D. C., May 15, 1958.

2. H. JefrreYs \& B. S. JefFreYs, Methods of Mathematical Physics, Third Edition, Cambridge University Press, Cambridge, 1956, p. 470.

3. Nat. BUR. Standards, Tables of Sine, Cosine and Exponential Integrals, v. 2, U. S. Government Printing Office, Washington 25, D. C., 1940.

4. WILLARI) EVAN BLEICK, Tables of Associated Sine and Cosine Integral Functions and of Related Complex-Valued l'unctions, Tech. Rep. No. 10, U. S. N., Bureau of Ships, Monterey, 1953. (RMT 1137, MTAC, v. 7, 1953, p. 242-243.)

5. M. MAsHIKo, 'T'ables of Generalized Exponential-, Sine- and Cosine Integrals Ei $(x+i y)$, Si $(x+i y), C_{i}(x+i y)$, Numerical Computation Bureau, Tokyo, Report No. 7, March, 1953. (RMT 1192, MT'AC, v. 8, 1954, p. 89.)

6. A. V. Hersher, Computing Programs for the Complex Exponential Integral, NAVORD Report No. 5909, NPG' Report No. 1646, June 1, 1959. 